\title{
¿Cómo promueven desafíos los padres a sus hijos? Estudio cualitativo con adolescentes de la Ciudad de Buenos fires
}

\author{
How Do Parents Promote Challenges to their Children? f Qualitative Study \\ with Adolescents from Buenos fires \\ Como promovem desafios os pais a seus filhos? Estudo qualitativo \\ com adolescentes da Cidade de Buenos fires
}

\author{
María Julia Raimundi, María Fernanda Molina, Lucía Bugallo* \\ Universidad de Buenos Aires, Argentina \\ Consejo Nacional de Investigaciones Científicas y Técnicas (CONICET)
}

Doi: dx.doi.org/10.12804/ap133.02.2015.06

\section{Resumen}

El objetivo del trabajo es presentar concepciones sostenidas por los adolescentes acerca de cómo sus padres promueven desafíos para su vida. Los desafíos son experiencias o situaciones que implican la superación de un obstáculo y conllevan el logro de un objetivo valioso para uno mismo. Los padres, como principales fuentes de socialización de los adolescentes, poseen un rol incuestionable para promover desafíos en ellos. Se realizaron 2 grupos focales y 12 entrevistas individuales. Participaron 27 adolescentes de entre 12 y 17 años de edad de una escuela de Buenos Aires (Argentina). Utilizando la Teoría Fundamentada, se encontraron 10 categorías de primer nivel y 3 categorías centrales para explicar aquellos aspectos que los adolescentes perciben que hacen sus padres para promoverles desafíos. Se logró un modelo conceptual para relacionar las categorías encontradas. El incentivo y el apoyo por parte de los padres son dimensiones fundamentales para la promoción de desafíos en los adolescentes. Asimismo, la presión y la falta de apoyo pueden obstaculizar su realización. Estudiar estas dimensiones familiares implica un aporte para comprender los factores que promueven un desarrollo positivo en la adolescencia.

Palabras clave: promoción de desafíos; padres; adolescentes; Teoría Fundamentada; desarrollo positivo.

* María Julia Raimundi, Consejo Nacional de Investigaciones Científicas y Técnicas (CONICET, Argentina), Facultad de Psicología, Universidad de Buenos Aires, Argentina, Universidad de Málaga, España; María Fernanda Molina, CONICET, Facultad de Psicología, Universidad de Buenos Aires; Lucía Bugallo, CONICET, Facultad de Psicología, Universidad de Buenos Aires.

Esta investigación fue financiada por el CONICET (Resolución 329 de 2011)/Secretaría de Ciencia y Técnica de la Universidad de Buenos Aires (Proyecto UBACyT 052. Directora: Dra. Nora Leibovich de Figueroa. Codirectora: Dra. Vanina Schmidt).

Se agradece a los alumnos, padres, docentes y autoridades de la escuela que participó en este estudio. Asimismo, se agradece a las licenciadas Mariel Giménez, Yésica Vargas, María Sottani, Karina Mignolo, Celina Selva, Pamela Parma, Silvana Cataldi y a Nicolás Robles López su colaboración en la recolección de datos.

La correspondencia relacionada con este artículo debe ser dirigida a María Julia Raimundi, CONICET, Facultad de Psicología, Universidad de Buenos Aires, Av. Independencia 3065 (1225) Buenos Aires, Ciudad Autónoma de Buenos Aires, Argentina. Correo electrónico:jraimundi@psi.uba.ar

Para citar este artículo: Raimundi, M. J., Molina, M. F. \& Bugallo, L. (2015). ¿Cómo promueven desafíos los padres a sus hijos?: Estudio cualitativo con adolescentes de la Ciudad de Buenos Aires. Avances en Psicología Latinoamericana, 33(2), 251-268. doi: dx.doi.org/10.12804/ap133.02.2015.06 


\section{fibstract}

The aim of this work is to present the conceptions held by adolescents about how their parents promote challenges in their life. Challenges are experiences or situations that involve overcoming an obstacle and lead to the achievement of a goal, valuable for oneself. Parents, as key socializing agents for adolescents, are an important source to promote these challenges. Two focus groups and twelve interviews were done with adolescents between 12 and 17 years, from a high school from Buenos Aires (Argentina). For data analysis, Grounded Theory was used. Ten open coding categories and three central categories were found to explain aspects that adolescents perceive their parents do to promote challenges to them. A conceptual model of the relations between categories is presented. Results indicate that incentive and support are fundamental dimensions of challenges' promotion in adolescents. In turn, pressure and lack of support can hinder the realization of the promotion of challenges. The description and comprehension of these family dimensions implies a greater understanding of factors that promote positive development in adolescence.

Key words: challenges' promotion; parents; adolescents; grounded theory; positive development.

\section{Resumo}

O objetivo do trabalho é apresentar concepções sustentadas pelos adolescentes acerca de como seus pais promovem desafios para sua vida. Os desafios são experiências ou situações que implicam a superação de um obstáculo e implica o sucesso de um objetivo valioso para si mesmo. Os pais, como principais fontes de socialização dos adolescentes, possuem um rol inquestionável para promover desafios nestes. Realizaram-se dois grupos focais e doze entrevistas individuais. Participaram 27 adolescentes entre 12 e 17 anos, de uma escola de Buenos Aires (Argentina). Empregando Teoria Fundamentada, encontraram-se dez categorias de primeiro nível e três categorias centrais para explicar aqueles aspectos que os adolescentes percebem que fazem seus pais para lhes promover desafios. Conseguiu-se um modelo conceitual para relacionar as categorias encontradas. O incentivo e o apoio por parte dos pais são dimensões fundamentais para a promoção de desafios nos adolescentes. Igualmente, a pressão e a falta de apoio podem obstaculizar sua realização. Estudar estas dimensões familiares implica um aporte para compreender os fatores que promovem um desenvolvimento positivo na adolescência.

Palavras chave: promoção de desafios; pais; adolescentes; Teoria Fundamentada; desenvolvimento positivo.

En las últimas décadas, la visión de la adolescencia como periodo problemático ha sido reemplazada por otra que se centra más en los aspectos positivos del desarrollo y la presentan como una etapa de evolución durante la cual el individuo se enfrenta a un amplio rango de demandas, conflictos y oportunidades. El adolescente contribuye positivamente a su propio desarrollo y se encuentra implicado en un proceso de negociación con sus padres, con objeto de ejercer un mayor control sobre su propia vida (Musitu, Buelga, Lila \& Cava, 2004).

De esta forma, la familia se encuentra en el deber de sincronizar dos movimientos antagónicos: la tendencia del sistema a la unidad, el mantenimiento de los lazos afectivos y el sentimiento de pertenencia y la tendencia hacia la diferenciación y la autonomía de sus miembros singulares (Musitu et al., 2004).

En el campo de la psicología, la entrada en el siglo XXI ha venido acompañada de distintas propuestas teóricas que han compartido un objetivo común: el desarrollo positivo de las personas (Balaguer, Castillo \& Duda, 2008). Diversos autores se han interesado por aquellas características de las personas y de sus entornos que promueven el bienestar y la satisfacción vital (e. g. Peterson, 2013; Sinnott, 2013) de las personas, en general, y de los adolescentes, en particular (e. g. Bassi, Steca, Monzani, Greco \& Delle Fave, 2013; Benson, Scales, Hamilton \& Sesman, 2006; Marques, Pais-Ribeiro \& Lopez, 2011). Debido a la importancia que posee 
la familia en esta etapa vital, resulta imprescindible incluirla al considerar el estudio de cualquier característica en la adolescencia.

En el contexto de este nuevo enfoque, la psicología positiva, surge una característica de la familia de particular importancia para el desarrollo de los adolescentes: la promoción de desafíos. Esta característica se refiere a la estimulación, la disciplina o el entrenamiento que los padres y otros miembros de la familia dan directamente al adolescente. Su propósito es fomentar la autonomía y la autodirección (Rathunde, Carroll \& Huang, 2000). Asimismo, incluye las expectativas que el adolescente percibe que su familia tiene hacia él y su deseo de cumplir con estas expectativas. Un ambiente familiar es desafiante cuando los padres esperan de sus hijos adolescentes que tomen grandes responsabilidades aprendan nuevas habilidades $\mathrm{y}$ tomen riesgos que los lleven a una mayor "individuación" (Rathunde et al., 2000). Dailey (2008) describe esta característica como comportamientos que brindan oportunidades para la estimulación y el desarrollo. Es decir, impulsan al adolescente para que ponga a prueba sus habilidades, lo cual permite que desarrolle, desde un rol activo, sus potenciales cognitivos, comportamentales, sociales $\mathrm{o}$ afectivos.

A su vez, los padres que promueven activamente desafíos en sus hijos los guían hacia el afrontamiento de tareas o situaciones difíciles, sin ser demasiado intrusivos ni dejándolos solos. De esta forma, estos padres proveen de experiencias a los adolescentes, que les brindan una sensación de competencia y dominio sobre las situaciones. Esto repercutirá en la percepción de control y actuará como barrera para el desarrollo de la ansiedad en la adultez (Zalta \& Chambless, 2011). Por lo tanto, el desafío promovido por los padres implica un recurso para el desarrollo positivo durante la adolescencia.

Numerosos estudios muestran la importancia del desafío promovido por los padres para el desarrollo positivo de los adolescentes, pues se asocia a diferentes indicadores de ajuste psicosocial en la adolescencia, como autoestima (Dailey, 2008; Schmidt \& Padilla, 2003), identidad (Dailey, 2008), implicación en actividades extracurriculares y rendimiento escolar (Schmidt \& Padilla, 2003), motivación intrínseca para realizar actividades de aventura (Lee, 2008) y focalización en metas importantes para el adolescente (Hektner, 2001; Rathunde et al., 2000; Rathunde, 2001). A su vez, estas características familiares dadas durante la adolescencia se asocian con el desarrollo de la creatividad (Gute, Gute, Nakamura \& Csikszentmihalyi, 2008) y la percepción de control en la adultez (Zalta \& Chambless, 2011).

Por otro lado, la promoción de desafíos por parte de los padres se ha incluido como una característica del entorno familiar que posibilita las experiencias óptimas de flow (fluir) en niños y adolescentes. Se ha definido el flow como un estado subjetivo en el que la persona está totalmente involucrada y hay un gran disfrute en la actividad que realiza en sí misma. Este estado se experimenta justamente cuando hay un equilibrio entre el desafío que plantea esta actividad y la habilidad de la persona (Csikszentmihalyi, 1990).

En un estudio realizado en Estados Unidos (Moon, 2003) se seleccionaron familias con alto apoyo y desafío, utilizando un cuestionario psicométrico, y luego se entrevistaron a los adolescentes que pertenecían a estas familias para indagar sobre las características de dicho entorno, en relación con una práctica particular, el fútbol. Estas familias se caracterizaban por tener una comunicación abierta y frecuente entre padres e hijos y pasar gran cantidad de tiempo juntos, específicamente, en el deporte practicado por el adolescente.

En otro estudio cualitativo con futbolistas universitarios (Lawrence, 2009), en el que se siguió la misma metodología que en el anterior, se encontró que en las familias con altos niveles de apoyo y promoción de desafíos, los padres están involucrados en las actividades recreativas que realizan los adolescentes fuera del hogar, manifiestan la 
importancia de la práctica de esa actividad y cada hijo tiene la libertad de elegir qué actividad hacer. Los adolescentes poseen metas orientadas hacia el futuro y los padres brindan apoyo hacia esas metas elegidas por el adolescente. En las familias que promueven desafío, pero hay poco apoyo, los padres raramente asisten a los partidos de fútbol, insisten en que el hijo puede hacerlo mejor, se pone el acento en el compromiso y el esfuerzo, existen discusiones sobre el financiamiento para practicar la actividad y se cuestionan las elecciones del hijo.

A pesar de que las investigaciones sobre el tema han resultado de gran utilidad para incorporar el desafío como una dimensión vinculada a indicadores de ajuste psicológico en la adolescencia, el concepto parece aún poco definido en relación con otros constructos y dimensiones familiares que se relacionan.

Asimismo, no queda clara cuál es la concepción que sostienen los mismos adolescentes sobre cómo sus padres pueden promoverles desafíos en sus vidas. Los últimos estudios citados aportan datos interesantes acerca de las características de las familias que promueven desafíos en sus hijos; sin embargo, esta característica fue identificada a priori, a través de un cuestionario y las entrevistas no se orientan a indagar qué es lo que realizan los padres para promoverles desafíos en diferentes ámbitos de su vida, sino que se centran en una actividad deportiva en particular.

Rodham, Brewer, Mistral y Stallard (2006) destacan la importancia de trabajar con las concepciones construidas por los mismos protagonistas, ya que la mirada del investigador puede sesgar aspectos importantes para comprender la conducta adolescente.

Conocer el significado de lo que es un desafío para los propios adolescentes brinda el cimiento inicial para poder indagar acerca de cómo los padres los promueven en ellos y la posibilidad de generar un constructo más claro y delimitado en relación con las demás dimensiones familiares.
Para indagar por el significado de la noción de desafío en esta etapa vital, en este mismo contexto sociocultural, Raimundi, Molina, Giménez y Minichiello (2014) realizaron un estudio cualitativo para comprender profundamente el significado de los desafíos en los propios protagonistas. Los adolescentes refirieron que los desafíos son experiencias o situaciones que implican la superación de un obstáculo o dificultad y conllevan el logro de un objetivo, valioso para uno mismo. Para realizar este desafío es necesario un esfuerzo y compromiso, y requiere poner en juego habilidades propias. $\mathrm{La}$ superación del desafío implica alcanzar el objetivo propuesto y, a su vez, sensaciones de superación personal y emociones positivas.

Los trabajos que se han dedicado a estudiar la promoción de desafíos por parte de los padres provienen en su totalidad del contexto anglosajón, específicamente de los Estados Unidos. Los significados que adoptan las evaluaciones familiares en un contexto sociocultural determinado son intransferibles a otros, debido a que las culturas imprimen sus propias formas (Schmidt et al., 2008).

Los adolescentes construyen su propia identidad y sus ideas acerca del futuro a partir de las distintas posibilidades que les ofrece el contexto en el que viven (Facio, Resett, Mistrorigo \& Micocci, 2006), y por ello las características de los entornos familiares y las interacciones entre padres e hijos deben ser consideradas construcciones históricas y sociales. Los estudios que se llevan a cabo teniendo tales particularidades aportan datos novedosos y permiten una indagación más profunda y sensible a la diferencias contextuales (Schmidt, Maglio, Messoulam, Molina \& Gonzalez, 2010).

La metodología cualitativa brinda la oportunidad de indagar acerca de las percepciones y experiencias de los grupos humanos sin restringir a los actores a las categorías preconcebidas o generadas en contextos muy distintos del propio investigador (Schmidt et al., 2008). 
Por lo tanto, el objetivo de este trabajo es presentar las concepciones que posee un grupo de adolescentes de la ciudad de Buenos Aires (Argentina) acerca de cómo sus padres promueven desafíos en ellos.

\section{Método}

Este trabajo forma parte de un proyecto de investigación más amplio que se orienta al estudio de los factores que influyen en las experiencias positivas de los adolescentes. Uno de los temas primordiales que se indagaron fueron las concepciones que poseen los adolescentes acerca de los desafíos y sus características (Raimundi et al., 2014). Por lo tanto, el presente trabajo constituye una continuación de este primer estudio.

Considerando el objetivo de esta investigación, se utilizó la metodología cualitativa, ya que permite restituir al individuo su cualidad de portador de una realidad social, una voz que no se restringe la propia experiencia, sino que logra ser representativa de una comunidad, de un medio social y de un tiempo histórico (Salguero, Córdoba \& Sapién, 2009).

Más específicamente, se utilizó el procedimiento de análisis de la Teoría Fundamentada (Glaser \& Strauss, 1967; Strauss \& Corbin, 1990), que implica que los hallazgos van emergiendo fundamentados en los datos recolectados, lo que permite el desarrollo de un conjunto de proposiciones teóricas.

Este trabajo se orientó al estudio de las concepciones de los adolescentes acerca de una temática específica. Llamamos concepciones a esas representaciones o conjunto de creencias, muchas de ellas implícitas o no del todo conscientes, que cumplen funciones predictivas y orientativas en las conductas humanas y que configuran las prácticas de las personas (Scheuer \& De la Cruz, 2010).

\section{Participantes}

Participaron 27 adolescentes (55.5\% varones), de entre 12 y 17 años de edad, que concurrían a una escuela secundaria privada de la ciudad de Buenos Aires (Argentina), con un nivel socioeconómico medio (más de la mitad de los padres tenían estudios universitarios). En la tabla 1 se describen las características de los adolescentes (sexo y edad) en función del instrumento de recolección de la información.

Tabla 1

Características de los participantes

\begin{tabular}{|c|c|c|c|c|c|}
\hline & \multicolumn{4}{|c|}{ Edad } & \multirow[b]{3}{*}{$\stackrel{0}{\ominus}$} \\
\hline & \multicolumn{2}{|c|}{ Entre 12 y 15 años } & \multicolumn{2}{|c|}{ Entre 15 y 18 años } & \\
\hline & $\begin{array}{c}\text { Mujeres } \\
n(\%)\end{array}$ & $\begin{array}{c}\text { Varones } \\
n(\%)\end{array}$ & $\begin{array}{c}\text { Mujeres } \\
n(\%)\end{array}$ & $\begin{array}{c}\text { Varones } \\
n(\%)\end{array}$ & \\
\hline Entrevistas & $3(25.0)$ & $3(25.0)$ & $3(25.0)$ & $3(25.0)$ & 12 \\
\hline $\begin{array}{l}\text { Grupo focal } \\
1\end{array}$ & $2(25.0)$ & $3(37.5)$ & $1(12.5)$ & $2(25.0)$ & 8 \\
\hline $\begin{array}{l}\text { Grupo focal } \\
2\end{array}$ & $1(14.3)$ & $3(42.8)$ & $2(28.6)$ & $1(14.3)$ & 7 \\
\hline Total & $6(40.0)$ & $9(60.0)$ & $6(50.0)$ & $6(50.0)$ & 27 \\
\hline
\end{tabular}

Con respecto a la composición familiar, la comunidad escolar que participó del estudio tenía un $63.6 \%$ de familias biparentales (ambos padres conviven con sus hijos), un $20.7 \%$ de familias monoparentales, un $7.1 \%$ de familias compuestas (incorporan otros no familiares) y un $8.7 \%$ posee otra conformación familiar. Estas características fueron evaluadas en un estudio más amplio.

La muestra fue seleccionada intencionalmente, por cuanto su propósito no fue la representatividad de los datos, sino la posibilidad de profundizar en el sentido de los fenómenos sociales (Maxwell, 2012). Teniendo en cuenta que la población de los adolescentes es muy amplia, se trabajó con adolescentes escolarizados y de clase media, por la accesibilidad a la muestra, considerando la edad de los participantes y trabajando con ambos sexos.

\section{Recolección de la información}

En virtud del objetivo de investigación los instrumentos de recolección de datos fueron: 
Entrevistas individuales. Se realizaron 12 entrevistas semiestructuradas que tuvieron una duración de 30 minutos, aproximadamente. Se indagó sobre las concepciones de los adolescentes acerca de los desafíos y los tipos de actividades que los propician, y específicamente, para explorar por la temática de este estudio (concepciones acerca de cómo sus padres promueven desafíos en ellos). Las preguntas guía fueron: ¿sentís que tus papás te alientan/animan a que te plantees desafíos? ¿Qué cosas/conductas/estrategias hacen tus papás para alentarte/animarte a asumir desafíos? ¿Podrías dar algún/algunos ejemplo(s)?

Grupos focales. Se realizó un grupo focal con ocho y otro con siete adolescentes, que no hubieran participado de las entrevistas individuales. Tuvieron una duración de 45 minutos, aproximadamente, y se utilizó la misma guía de preguntas que en las entrevistas. En este caso, el propósito fue tomar conocimiento del sentido dado a los desafíos desde la perspectiva adolescente, pero a partir de una elaboración grupal. Esto posibilita, a su vez, la triangulación de la información, lo que otorga mayor credibilidad al estudio.

\section{Procedimiento}

Se estableció el contacto con la escuela, se explicó a las autoridades los objetivos del estudio y se entregaron las notas de consentimiento informado para que los padres firmaran el permiso para la participación de sus hijos en la investigación.

Los adolescentes fueron seleccionados al azar mediante las notas de consentimiento entregadas al equipo de investigación, teniendo en cuenta una distribución equiparada por curso y por sexo, y se los invitó a participar. La participación de todos los adolescentes fue voluntaria y se cuidaron las condiciones de privacidad, a fin de facilitar que los participantes pudieran expresarse libremente.
Al comenzar, se hizo hincapié en la confidencialidad de los datos y se presentó el objetivo general de la investigación. Las sesiones de grupos focales y las entrevistas individuales fueron grabadas.

El formato del grupo focal fue establecido siguiendo la propuesta de Krueger (1991). Los grupos fueron conducidos por un moderador y participaron dos asistentes, quienes registraron el comportamiento no verbal y agregaron comentarios y síntesis, cuando lo consideraron necesario. Al finalizar cada sesión, tanto el moderador como el asistente presentaron, de manera resumida, los principales puntos que los(as) participantes habían tratado, y se dejó un espacio para preguntas que pudieran surgir en esa instancia.

\section{Análisis de datos}

En primer lugar, se transcribieron las grabaciones de los grupos focales y entrevistas. Se transcribió tanto lo comunicado verbalmente como las anotaciones del comportamiento no verbal. Cada uno de los integrantes del equipo de investigación recibió una copia de las transcripciones y, de modo independiente, se identificaron las unidades de significado relevantes para el objetivo de investigación, asignándoles un código (codificación abierta o de primer nivel).

Se estableció un libro de códigos que implicó el desarrollo de reglas explícitas para la lectura del material. De esta forma, quedó explicitado cómo segmentar el corpus y el modo en que se registraron los datos. Con base en las categorías propuestas, se confeccionaron fichas de análisis en las que se consignó una palabra clave o un juicio extraído del texto en su literalidad. Luego se compararon las unidades de significado y la descripción de las categorías realizada por cada integrante del grupo y se mantuvieron aquellas resultantes del consenso entre los investigadores, tomando como criterios su fácil inferencia y aplicación y su relevancia teórica. 
Piñuel Raigada (2002) sugiere que el trabajo con las fichas de análisis debe implicar a un equipo de colaboradores, los "procesadores de datos", quienes analizan las categorizaciones realizadas, con el fin de interpretar los datos de segundo orden que sirven para exponer y describir las conclusiones del estudio. Tres investigadores, de forma independiente, reelaboraron las categorías y se adoptó la agrupación surgida del consenso.

Todo el procedimiento se orientó a establecer la credibilidad, la dependencia y la confirmabilidad de los datos (Hernández Sampieri, Fernández-Collado \& Baptista Lucio, 2010), como criterios de rigor en la investigación cualitativa, a fin de aportar mayor validez al proceso realizado. Se estableció una consistencia lógica de los datos, es decir, el grado en que diferentes investigadores que recolectan datos similares y efectúan los mismos análisis, generan resultados equivalentes.

Por último, siguiendo los lineamientos propuestos para la Teoría Fundamentada, se establecieron relaciones conceptuales entre las categorías emergidas de los datos, lo que dio como resultado un modelo respecto de la promoción de desafíos por parte de los padres desde la percepción de los adolescentes de este estudio (figura 1). Esta reelaboración de categorías y construcción del modelo permitió identificar tres categorías centrales, que organizan las categorías de primer nivel, captando el tema de estudio en su complejidad. A su vez, para lograr una mayor comprensión y ordenamiento de los datos se introdujeron categorías intermedias o de segundo nivel, que vinculan las categorías de primer nivel con las centrales (Coleman \& Unrau, 2005, citado en Hernández Sampieri et al., 2010).

\section{Resultados}

En la tabla 2 se presenta un resumen de las categorías centrales y de primer nivel, con ejemplos de fragmentos textuales de los adolescentes.
Tabla 2

Categorías centrales, de primer nivel y ejemplos

\begin{tabular}{|c|c|c|}
\hline $\begin{array}{l}\text { Categoría } \\
\text { central }\end{array}$ & $\begin{array}{l}\text { Categoría de } \\
\text { primer nivel }\end{array}$ & Ejemplos \\
\hline $\begin{array}{l}\text { I. Promoción } \\
\text { de los padres } \\
\text { para que los } \\
\text { adolescentes } \\
\text { encuentren } \\
\text { desafíos }\end{array}$ & $\begin{array}{l}\text { I.1. Incen- } \\
\text { tivo para la } \\
\text { realización de } \\
\text { actividades } \\
(n=11)\end{array}$ & $\begin{array}{l}\text { "generalmente me } \\
\text { alientan a que tenga } \\
\text { un objetivo. Dándome } \\
\text { actividades, proponien- } \\
\text { do items a cumplir" } \\
\text { (varón, } 14 \text { años) }\end{array}$ \\
\hline \multirow{6}{*}{$\begin{array}{l}\text { II. Promoción } \\
\text { de los padres } \\
\text { para que los } \\
\text { adolescentes } \\
\text { realicen los } \\
\text { desafíos }\end{array}$} & $\begin{array}{l}\text { II.2. Acompa- } \\
\text { ñamiento } \\
(n=8)\end{array}$ & $\begin{array}{l}\text { "el hecho de estar todo } \\
\text { el tiempo ahí, aunque } \\
\text { no los necesite, sabés } \\
\text { que ellos están. Si jugás } \\
\text { bien, te felicitan, y si } \\
\text { jugás mal, también" } \\
\text { (mujer, } 15 \text { años) }\end{array}$ \\
\hline & $\begin{array}{l}\text { II.3. Brindar } \\
\text { un espacio para } \\
\text { hablar }(n=10)\end{array}$ & $\begin{array}{l}\text { "siempre hay un espa- } \\
\text { cio de charla familiar } \\
\text { y me preguntan cómo } \\
\text { estoy, si me siento } \\
\text { cómodo, si está todo } \\
\text { bien" (varón, } 16 \text { años) }\end{array}$ \\
\hline & $\begin{array}{l}\text { II.4. Apuntala- } \\
\text { miento }(n=3)\end{array}$ & $\begin{array}{l}\text { "Sí, me proponen de- } \\
\text { safíos, lo que hacen es } \\
\text { decirme que estoy ca- } \\
\text { pacitada para afrontar } \\
\text { esos desafios" (mujer, } \\
17 \text { años) }\end{array}$ \\
\hline & $\begin{array}{l}\text { II.5. Consejos } \\
\text { prácticos o téc- } \\
\text { nicos }(n=7)\end{array}$ & $\begin{array}{l}\text { "me dan consejos de } \\
\text { cómo estudiar, alguna } \\
\text { palabrita y esas cosas, } \\
\text { sí me sirve mucho" } \\
\text { (varón, } 16 \text { años) }\end{array}$ \\
\hline & $\begin{array}{l}\text { II.6. Apoyo } \\
\text { material }(n=7)\end{array}$ & $\begin{array}{l}\text { "Me dan lo que nece- } \\
\text { sito para las cosas que } \\
\text { hago. Por ejemplo, me } \\
\text { compraron un pen drive } \\
\text { para trabajar en el pro- } \\
\text { yecto" (varón, } 16 \text { años) }\end{array}$ \\
\hline & $\begin{array}{l}\text { II.7. Valoración } \\
\text { del apoyo brin- } \\
\text { dado }(n=3)\end{array}$ & $\begin{array}{l}\text { "Y creo que ellos me } \\
\text { dan todo y se re preocu- } \\
\text { pan por mí, y eso tam- } \\
\text { bién es como plantear- } \\
\text { me que por el esfuerzo } \\
\text { que hacen ellos hay que } \\
\text { seguir y eso creo que } \\
\text { puede ser" (mujer, } 17 \\
\text { años) }\end{array}$ \\
\hline
\end{tabular}




\begin{tabular}{|c|c|c|}
\hline $\begin{array}{l}\text { Categoría } \\
\text { central }\end{array}$ & $\begin{array}{l}\text { Categoría de } \\
\text { primer nivel }\end{array}$ & Ejemplos \\
\hline $\begin{array}{l}\text { II. Promoción } \\
\text { de los padres } \\
\text { para que los } \\
\text { adolescentes } \\
\text { realicen los } \\
\text { desafíos }\end{array}$ & $\begin{array}{l}\text { II.8. Padres } \\
\text { como modelos } \\
(n=2)\end{array}$ & $\begin{array}{l}\text { "Y también está bueno } \\
\text { que te acompañen, por- } \\
\text { que si fueran dejados, } \\
\text { por ahi yo también de- } \\
\text { jaría" (mujer, } 15 \text { años) }\end{array}$ \\
\hline \multirow[b]{2}{*}{$\begin{array}{l}\text { III. Aspectos } \\
\text { que obstacu- } \\
\text { lizan la rea- } \\
\text { lización de } \\
\text { desafíos }\end{array}$} & $\begin{array}{l}\text { III.9. Presión } \\
(n=4)\end{array}$ & $\begin{array}{l}\text { "por ahí llega a ser } \\
\text { presión para que hagas } \\
\text { lo que ellos quieren o lo } \\
\text { que consideran mejor } \\
\text { para vos" (mujer, } 14 \\
\text { años) }\end{array}$ \\
\hline & $\begin{array}{l}\text { III.10. Falta de } \\
\text { apoyo }(n=3)\end{array}$ & $\begin{array}{l}\text { "a mí me molesta que } \\
\text { tal vez... mi mamá to- } \\
\text { do el tiempo me alienta } \\
\text { más al estudio. Cual- } \\
\text { quier cosa, antes el } \\
\text { estudio... Y cualquier } \\
\text { cosa que te guste igual, } \\
\text { todo te lo echa en cara. } \\
\text { 'Ay no, pero estás ha- } \\
\text { ciendo esto, pero nada } \\
\text { del estudio"” (mujer, } 16 \\
\text { años) }\end{array}$ \\
\hline
\end{tabular}

\section{Promoción de los padres para que los adolescentes encuentren desafíos}

Esta primera categoría central hace referencia al incentivo que los adolescentes reciben por parte de sus padres para que encuentren o se propongan desafíos.

\section{I.1. Incentivo para la realización de activida-} des que pueden constituir un desafío. Los padres presentan o proponen al adolescente actividades para que realice (deportes, actividades creativas, etc.) y luego el adolescente la(s) elige y continúa realizándola(s) porque le gusta o disfruta con ella(s). Aunque hay otras personas que realizan esta tarea, los padres aparecen como las principales figuras.

"Sí, me proponen los desafios para que tenga que elegir" (varón, 16 años).
"Ellos me dijeron, ¿no querés ir los sábados y los domingos a practicar fútbol? Y desde ahí que fui" (varón, 15 años).

Algunos adolescentes perciben diferencias entre las actividades que les proponen su padre y su madre, y otros adolescentes no perciben estas diferencias. En ocasiones las actividades propuestas por los padres pueden estar basadas en su experiencia personal (por ejemplo: haber sido profesores o haber practicado ese deporte). Otros opinan que sus padres pueden priorizar la realización de unas actividades sobre otras.

"A mi papá le gusta mucho que yo haga deportes porque él fue maestro de taekwondo... A mí y a mi hermano nos incentiva, digamos, y a mi mamá... le gusta más lo social, que sea más social, que salga, que en el colegio no me junte siempre con las mismas personas, que sociabilice más" (mujer, 14 años).

"A mí me dicen: primero que estudie, después que vaya al club" (varón, 14 años).

\section{Promoción de los padres para que los adolescentes realicen los desafíos}

La segunda categoría central comprende siete categorías de primer nivel que constituyen diferentes formas en que los padres promueven que los adolescentes realicen los desafíos que se proponen.

II.2 Acompañamiento. Los padres promueven que los adolescentes realicen sus desafíos mediante la presencia física o afectiva (e.g. desearles suerte), acompañándolos en las actividades o situaciones que los adolescentes consideran un desafío.

"me apoyaron, estuvieron conmigo... Sentimentalmente, porque yo me pongo mal si no me salen las cosas. Entonces me decían que ya me iba a salir, me apoyaron de esa forma" (mujer, 15 años).

"me cuidan, dan consejos [...]. Me desean suerte" (varón, 14 años). 


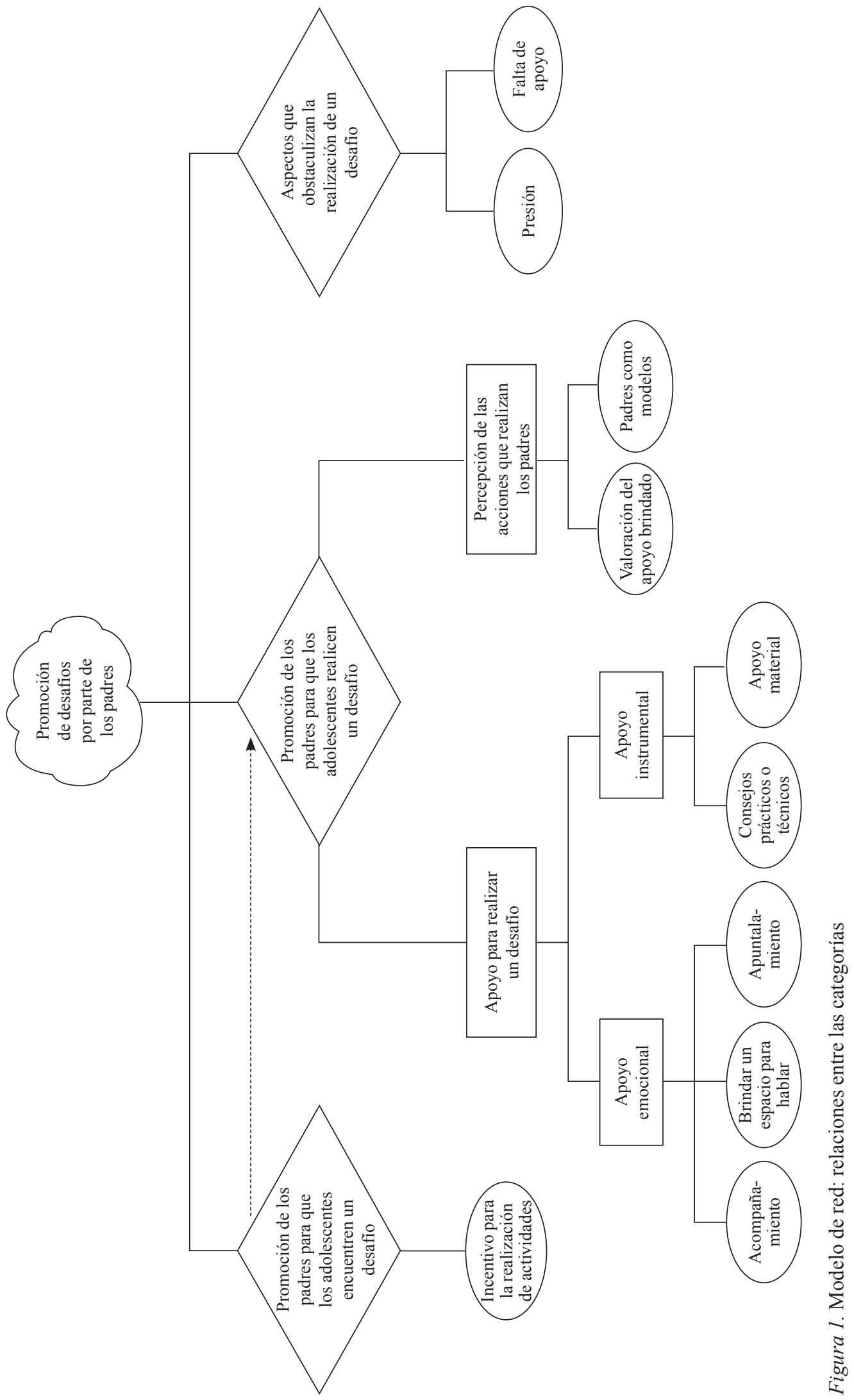


II.3 Brindar un espacio para hablar. La posibilidad de ser escuchado por sus padres respecto de las actividades o situaciones que implican un desafío para los adolescentes. Ellos identifican espacios, como "la charla familiar" donde pueden hablar de lo que les pasa y sienten que son apoyados.

"Están siempre, me escuchan, les puedo contar lo que sea, que ellos siempre me escuchan" (mujer, 15 años).

“... y bueno este año tuve novio y como que siempre me apoyaban, me decían, contanos qué es para vos tener novio... y como que era un desafio tener novio" (mujer, 14 años).

II.4 Apuntalamiento. El diccionario define el verbo apuntalar como "sostener, dar firmeza" (Espasa Calpe, 2005). Los padres brindan confianza sobre la capacidad para afrontar los desafíos que el adolescente tiene por delante y brindan el apoyo necesario para no abandonar esta tarea cuando se vuelve difícil. Algunas veces ese apuntalamiento implica una acción directa por parte de los padres sobre la conducta del adolescente que involucra la puesta de límites, por ejemplo, interrumpir una actividad (e. g. apagar la computadora) para que el adolescente se avoque a la actividad que constituye su desafío.

"Te dicen lo que tenés que hacer. Pero a veces no les das bola y te tienen que sacar la computadora o algo como para tomártelo en serio [...] Entonces me pongo las pilas y me pongo a estudiar" (mujer, 13 años).

"sí, yo creo que cuando me apoyan y no me dejan aflojar a veces cuando estoy muy cansada o estresada es una manera de ayudarme a plantearme nuevos desafios, aunque sean cosas chiquitas [...]Y cuando estoy mal que quiero aflojarle a algo, lo que hacen es recordarme cómo la peleé anteriormente y también remarcarme todo lo que está en juego, como que intentan tranquilizarme y que yo vea las cosas más en frío" (mujer, 17 años).
II.5 Consejos prácticos o técnicos. Se trata de consejos específicos que ayudan al adolescente a llevar a cabo sus actividades o situaciones desafiantes.

"Lo charlé con mis papás, me dijeron que no le diera bolilla porque es un chico malo, bueno después me dijeron que le dijera esto que a mí no me gusta que me insulten, fui yo y le dije" (mujer, 12 años).

II.6 Apoyo material. Los padres brindan recursos materiales para que el adolescente pueda realizar las actividades que asume como desafíos. Más específicamente, se refiere a los traslados a la actividad y el brindar los materiales o el dinero para poder realizarla.

"Me llevan a todos lados, no sé, me tengo que levantar a las siete de la mañana y ellos se levantan, se ponen el despertador, y me acompañan. En vez de mandarme, no sé, en un micro por ejemplo, me llevan en el auto" (mujer, 15 años).

\section{II.7. Valoración del apoyo brindado por los} padres. Se trata de la percepción y valoración del apoyo brindado por los padres que incentiva y motiva en la persistencia en las actividades que ellos se han planteado como desafiantes.

"Mis padres se sacrifican para poder pagarme este colegio, hacen un sacrificio muy grande, y bueno, yo trato de responderles con notas porque, pónele, ellos no pueden darse algunos lujos o comprarse ropa y demás para poder pagarme el estudio, entonces yo les quiero corresponder con notas. Entonces mi objetivo, mi desafio es tener un buen rendimiento académico y funcionar bien" (varón, 16 años).

II.8 Padres como modelos. Muchas veces los padres aparecen como modelos para los adolescentes, lo que implica un incentivo para realizar la actividad que los adolescentes se han planteado como desafío. 
"Se basan en experiencias personales para decirme que ellos a través del esfuerzo, pudieron superar el desafio" (mujer, 17 años).

\section{Aspectos que obstaculizan la realización de desafíos}

Por último, la tercera categoría central implica dos aspectos referidos por los adolescentes que pueden obstaculizar la realización de desafíos.

\section{III.9 Presión para realizar actividades que no} son un desafío. Los adolescentes pueden percibir que los padres los presionan para que hagan lo que los padres quieren o consideran que es lo mejor para el adolescente. Lo sienten como una presión porque se trata de actividades que no suponen un desafío para ellos.

“... mi papá tenis. A mí me gusta, pero llega un momento que le tengo, le quiero decir 'no, no quiero hacer tenis pero quiero otra cosa" (mujer, 13 años).

"Si te lo ofrecen, que no lo hagas porque tengas presión, que lo hagas porque te gusta" (varón, 12 años)

\section{III.10 Falta de apoyo para realizar activida-} des que son un desafío. Los adolescentes perciben una falta de apoyo para las actividades que ellos consideran que son un desafío o las consideran importantes. A veces, esta falta de apoyo se vincula con que los padres priorizan otras actividades sobre las que el adolescente elije.

"Ponele, toda tu vida tus viejos fueron abogados. Yo, en mi caso, quiero estudiar Letras. La respuesta, cada vez que digo Letras... es muy gracioso... “¿Cómo Letras? Pero, ¿a qué te vas a dedicar? Pero, ¿Letras? ¿No querés mejor Abogacía? [...] o sea, todo bien que te apoyen... o por ahi ellos piensan que te apoyan ... pero no" (mujer, 17 años).

\section{Modelo de red: relaciones entre las categorías}

La utilización de Teoría Fundamentada en el análisis de datos se orienta a la construcción de una teoría como resultado y no solamente un conjunto de enunciados descriptivos (Suárez Relinque, del Moral Arroyo \& González Fernández, 2013). Para ello, se elaboró un modelo de red (figura 1) que permite encontrar sentido y significado a las relaciones entre los temas y categorías emergidas del análisis (Hernández Sampieri et al., 2010).

En la figura, los óvalos identifican las categorías de primer nivel (emergidas de los dichos de los adolescentes entrevistados), los rombos identifican las categorías centrales (es decir, globales que permiten establecer relaciones entre las categorías de primer nivel y construir un modelo) y los rectángulos señalan las categorías intermedias o de segundo nivel, que ordenan y vinculan las categorías de primer nivel con las centrales.

El modelo muestra las concepciones de los adolescentes entrevistados acerca de lo que realizan sus padres para promover desafíos en ellos.

Por un lado, el incentivo a la realización de actividades que pueden constituir un desafío para ellos, que implica acciones o conductas de los padres que promueven que los adolescentes encuentren desafíos en sus vidas, y por el otro, el apoyo para poder realizar o afrontar esos desafíos. Este apoyo engloba las categorías de primer nivel de acompañamiento, brindar un espacio para hablar y apuntalamiento, que tienen que ver con el apoyo de tipo emocional o afectivo (categoría intermedia o de segundo nivel), y el apoyo instrumental (categoría intermedia), que implica las categorías de primer nivel de consejos prácticos y el apoyo material. Por otro lado, la percepción de acciones que realizan los padres (categoría intermedia), como el hecho de valorar ese apoyo recibido y tomar a los padres como modelos, puede promover la persistencia y esfuerzo de los adolescentes para realizar desafíos. 
Puede existir una relación entre el incentivo y el apoyo brindado por los padres, aunque dicha relación no se presenta necesariamente: los adolescentes perciben que a partir de que los padres ofrecen actividades o experiencias que pueden constituir un desafío, ellos lo asumen y luego reciben el apoyo necesario para poder llevarlo a cabo. Por ejemplo: “mi mamá me dijo que estaría bueno que yo cocine [...] y me pareció una buena idea porque para mí es nuevo, nunca fui a un lugar de cocina, yo dije que me pareció una buena idea y me dijo: bueno, yo te voy a llevar" (mujer, 12 años).

Por último, los adolescentes identifican aspectos que pueden obstaculizar la realización de desafíos: la presión que sus padres pueden ejercer sobre ellos para que realicen lo que ellos consideran importante y la falta de apoyo percibida para la realización de lo que el adolescente elige como desafío.

\section{Discusión}

El objetivo de este trabajo fue indagar las concepciones de un grupo de adolescentes acerca de lo que realizan sus padres para promover desafíos en ellos.

Los resultados indican que, desde la perspectiva de los adolescentes entrevistados, hay dos formas en que los padres promueven desafíos. Por un lado, el incentivo brindado para que los adolescentes encuentren desafíos. Los padres son las principales figuras que proponen y muestran actividades para que los adolescentes realicen (como deportes o actividades creativas) y luego son estos los que continúan realizándolas porque disfrutan con ellas. La literatura muestra que el aliento o ánimo brindado por los padres es uno de los principales predictores de implicación en actividades físicas (Biddle \& Goudas, 1996; Edwardson \& Gorely, 2010) y actividades extracurriculares en general (Anderson, Funk, Elliott \& Smith, 2003).

Por otro lado, los padres promueven la realización de los desafíos que los adolescentes se proponen, brindando su apoyo. Este apoyo posee dos vertientes: la dimensión emocional o afectiva y la dimensión instrumental. La primera se refiere al acompañamiento, espacios para hablar y acciones de "apuntalamiento" que los adolescentes perciben que les brindan sus padres para guiarlos en la realización del desafío. La dimensión instrumental implica los consejos prácticos o técnicos y el apoyo material.

Ambas vertientes han sido destacadas en los estudios sobre apoyo social en adolescentes, señalando que la familia constituye un entorno fundamental para su desarrollo y ajuste psicosocial (e.g. Minichiello, 2009; Musitu et al., 2004). Particularmente, el apoyo de los padres adquiere un rol principal en la implicación inicial en actividades extracurriculares como deportes, voluntariados, música o danza (Anderson et al., 2003) y en la intensidad de la realización de actividad física (Edwardson \& Gorely, 2010). Este apoyo se desarrolla a través de conductas como el transporte, ir a ver a los hijos a sus prácticas, hacer actividad física con ellos, y en la importancia atribuida a la actividad. En el estudio cualitativo de Lawrence (2009) se muestra que en las familias identificadas como de alto apoyo y desafío, cada hijo tiene la libertad de elegir qué actividad hacer y los padres se interesan y están involucrados en la práctica de esa actividad elegida por el adolescente.

Asimismo, el apoyo emocional e instrumental es la conducta positiva más destacada por tenistas adolescentes en el desarrollo de su talento (Lauer, Gould, Roman \& Pierce, 2010) y se asocia al disfrute y autoestima, tanto en varones como en mujeres que practican este deporte (Leff \& Hoyle, 1995).

Desde la Teoría de la Autodeterminación (Ryan \& Deci, 2000) y la Teoría de las Metas de Logro (Ames, 1992; Dweck, 1999) se plantea que el contexto social que rodea a los adolescentes (e.g., padres, compañeros, profesores) puede intervenir significativamente en la forma en la que los jóvenes viven la experiencia en un contexto determinado (escolar, deportivo, etc.), ya que los otros significativos adquieren un rol determinante en el 
compromiso y disfrute de la actividad que realiza el adolescente (Duda, 2013). De esta manera, los estudios empíricos han mostrado que un clima orientado a la tarea, es decir, un contexto cuyo interés se centra en adquirir maestría a través del esfuerzo y la percepción de la habilidad es autorreferenciada (Duda, 2013), predice una mayor motivación autodeterminada y experiencia óptima (Moreno, Cervelló \& González-Cutre, 2010) y una menor intención de abandono en el deporte (Quested et al., 2013).

Los desafíos son experiencias o situaciones difíciles que el adolescente se plantea afrontar y que, una vez alcanzados, producen sensaciones positivas de logro y autosuperación. Muchas de las estas situaciones de desafío son planteadas en los escenarios de las actividades estructuradas como el deporte y las actividades artísticas o creativas (Raimundi et al., 2014). Por lo tanto, contar con el apoyo emocional y el apoyo material e instrumental de los padres, permite que el adolescente se involucre y desarrolle sus habilidades a través del proceso de superación de los desafíos que se le van plateando en estas actividades.

Además del apoyo, otra de las formas en que los padres promueven la realización de desafíos en sus hijos es a través de la percepción que los adolescentes tienen de ellos y de la forma en que los apoyan para la realización de estos desafíos.

Los padres pueden ser modelos de los adolescentes, por cuanto muestran conductas y, a la vez, las consideran importantes para que los adolescentes las realicen. Por ejemplo, en la revisión de Edwardson y Gorely (2010), el nivel percibido de actividad física de los padres es uno de los predictores del involucramiento e intensidad de la actividad del adolescente.

Rathunde (2001), autor pionero en el estudio de la promoción de desafíos, plantea que los padres que promueven desafíos son percibidos por sus hijos como modelos de comportamientos autodirigidos y esta característica contribuye al logro de la autonomía y el desarrollo.
Además del apoyo emocional e instrumental, se encontró en el presente estudio que la valoración de ese apoyo también es percibida como un aspecto que sostiene al adolescente en la realización de sus desafíos. Desde el modelo de evaluación cognitiva de Lazarus y Folkman (1984) esta valoración, tanto la del apoyo como la de los padres como modelos, podría ser interpretada como un recurso que posee el adolescente a la hora de asumir un desafío, y que le permite movilizar las estrategias de afrontamiento en pos del logro de este objetivo, valioso para él mismo.

Por lo tanto, se trata de aspectos novedosos que se deben tener en cuenta a la hora de pensar en estrategias para incentivar a los adolescentes, desde el rol parental, a fin de que puedan involucrarse en actividades que pueden constituir un desafío.

Por otro lado, los adolescentes han identificado aspectos que pueden obstaculizar la realización de desafíos. Por un lado, pueden sentir presión para realizar actividades que los padres consideran que son importantes pero ellos no y, por el otro, pueden percibir falta de apoyo para las actividades que estos últimos consideran son un desafío.

Los estudios muestran consistentemente que la presión ejercida por los padres en las actividades extracurriculares de sus hijos adolescentes se asocia a menos actividades realizadas por estos, menor disfrute en actividades artísticas y deportivas (Anderson et al., 2003) y menor compromiso con el deporte (Torregrosa et al., 2007). La presión está relacionada con un desequilibrio entre el aliento o ánimo que brindan los padres y lo que desean los adolescentes en sus actividades, que produce una percepción de pérdida de control sobre la actividad, quedando su dirección en manos de sus padres (Lee $\&$ Maclean, 1997). Por otro lado, tiene que ver con expectativas de éxito, críticas y reacciones en las derrotas del adolescente (Leff \& Hoyle, 1995).

En los adolescentes del presente estudio, la presión se relaciona con la exigencia en la realización de actividades que para el adolescente no constituyen un desafío y, por lo tanto, no encuentra 
motivación para realizarlas. Un desafío implica una meta, algo a lo que el adolescente se orienta, y siempre es algo importante para él mismo (Raimundi et al., 2014). Por lo tanto, si el adolescente no percibe esa importancia y solo es incentivado desde afuera, la actividad nunca podrá convertirse en un desafío.

Con respecto a la falta de apoyo para la realización de actividades que sí constituyen un desafío para el adolescente, se puede concluir que si el apoyo de los padres es un recurso fundamental para la realización de los desafíos, su falta implica un obstáculo a la hora de afrontarlos. Este aspecto fue identificado de esta forma por los adolescentes del presente estudio.

Muchos trabajos han mostrado que la promoción de desafíos por parte de los padres es una característica asociada al desarrollo positivo de los adolescentes (Csikszentmihalyi, Rathunde \& Whalen, 1993; Dailey, 2008; Hektner, 2001; Rathunde et al., 2000; Rathunde, 2001); sin embargo, las definiciones del constructo no especifican ni describen en profundidad cuáles serían las conductas que realizan los padres para generar esta promoción de desafíos en sus hijos adolescentes.

Este trabajo se orientó a dar luz sobre cuáles son las concepciones de un grupo de adolescentes acerca de lo que realizan sus padres para promover desafíos en ellos. Esta puesta en marcha de desafíos implica un medio para un desarrollo positivo en la adolescencia; por lo tanto, allí radica la importancia de su conocimiento y comprensión.

La metodología utilizada se orientó a ganar en profundidad conceptual y validez ecológica, y esto mismo, que caracteriza lo cualitativo, impide generalizar los resultados sobre un grupo mayor de personas. Actualmente se considera que los métodos cualitativos y los cuantitativos son herramientas complementarias de indagación, que, al articularse, permiten un conocimiento más integral sobre la dinámica social que se quiere abordar (Velandia Morales, 2008). En el futuro, se espera contar con más estudios acerca de esta temática, con la implementación de diversas metodologías que se complementen en el abordaje de este fenómeno social.

Con respecto a los participantes, este trabajo fue realizado con adolescentes de una escuela secundaria privada de la ciudad de Buenos Aires. Esto implica que los datos deben restringirse al entendimiento de este fenómeno en un contexto con características sociodemográficas particulares. Como perspectiva futura de este estudio, podría ser interesante comparar las concepciones que sostienen adolescentes de otros contextos socioeconómicos y socioculturales. ¿Existirán diferencias en las concepciones que realizan los adolescentes en función del nivel socioeconómico o cultural? Estudios anglosajones (e.g. Anderson et al., 2003) muestran que la cantidad de actividades extracurriculares en las que participan los adolescentes está asociada al nivel socioeconómico familiar. Por lo tanto, ¿qué diferencias podrá haber en la percepción de la promoción de desafíos por parte de los padres el tener el acceso a la realización de actividades extracurriculares como los deportes o las actividades artísticas? Asimismo, podrían darse diferentes formas de incentivo y apoyo en función de los diferentes tipos de desafíos con los que los adolescentes se encuentran (Raimundi et al., 2014), por lo que es indispensable seguir indagando en este sentido.

Por otro lado, sería interesante profundizar acerca de la existencia de diferencias por edad o por género en los tipos de incentivos y apoyo por parte de los padres a la hora de asumir un desafío para los adolescentes.

Este estudio ha indagado sobre la promoción de desafíos por parte de los padres desde la perspectiva de los hijos adolescentes. En el futuro, sería interesante explorar acerca de la percepción de los padres sobre las conductas o acciones que realizan, ya que podría haber discrepancias entre lo percibido por los adolescentes y sus padres. Asimismo, es importante indagar, por un lado, por el papel diferencial que podrían tener las madres y los padres de los adoles- 
centes en esta característica asociada al desarrollo positivo y, por el otro, si existen diferencias en función de la composición familiar del adolescente. Se ha mostrado que para algunas características del funcionamiento familiar en nuestro contexto $(e . g$. comunicación, cohesión, flexibilidad) los padres ejercen influencias diferentes en el desarrollo de los adolescentes (Schmidt et al., 2010).

\section{Implicaciones prácticas del estudio}

El compromiso con un desafío implica un camino hacia la autosuperación y el logro de metas que el adolescente se plantea como importantes para sí mismo. A su vez, la posibilidad de percibir desafíos en las actividades que el adolescente realiza puede llevarlo a experiencias óptimas de disfrute, como la experiencia subjetiva de flow.

Este estudio contribuyó al conocimiento de lo que adolescentes perciben que realizan sus padres para incentivar la asunción y realización de desafíos. Esto puede implicar un aporte para los profesionales, padres y educadores que trabajan con adolescentes de nuestro medio, para contribuir a su bienestar y desarrollo positivo.

Conocer el sentido y significado atribuido por los propios protagonistas en un contexto sociocultural específico permite una comprensión más profunda del constructo en su complejidad y supera la limitación de aproximaciones sesgadas por la visión adulta del comportamiento adolescente (Rodham et al., 2006).

Son muchos los agentes y las instituciones que desempeñan un papel en el desarrollo de los niños: la familia, los pares, la escuela, los medios de comunicación, etc. A medida que van entrando en la adolescencia, las interacciones sociales en las que los jóvenes participan se incrementan en cantidad y complejidad. Sin embargo, la familia sigue siendo el contexto más importante en el que se dirimen las influencias socializadoras, debido a que las influencias familiares son las primeras y las más persistentes, y además, las relaciones familiares se caracterizan por una especial intensidad afectiva y capacidad configuradora sobre las relaciones posteriores fuera de la familia (Muñoz Silva, 2005).

Conocer profundamente aquellos factores que permiten que los adolescentes se desarrollen en todo su potencial, puede ser una clave a la hora de pensar la educación de los jóvenes con el foco orientado al logro de una salud integral.

\section{Referencias}

Ames, C. (1992). Achievement goals and adaptive motivational patterns: The role of the environment. In G. C. Roberts (Ed.), Motivation in sport and exercise (pp. 161-176). Champaign, IL: Human Kinetics.

Anderson, J., Funk, J., Elliott, R. \& Smith, P. (2003). Parental support and pressure and children's extracurricular activities: Relationships with amount of involvement and affective experience of participation. Journal of Applied Developmental Psychology, 24(2), 241-257. doi:10.1016/S0193-3973(03)00046-7

Balaguer, I., Castillo, I. \& Duda, J. (2008). Apoyo a la autonomía, satisfacción de las necesidades, motivación y bienestar en deportistas de competición: un análisis de la teoría de la autodeterminación. Revista de Psicología del Deporte, 17(1), 123-139.

Bassi, M., Steca, P., Monzani, D., Greco, A. \& Delle Fave, A. (2013). Personality and optimal experience in adolescence: Implications for well-being and development. Journal of Happiness Studies, 14(3), 1-15. doi:10.1007/s10902013-9451-x

Benson, P., Scales, P., Hamilton, S. \& Sesman, A. (2006). Positive youth development: Theory, research and applications. In R. Lerner (Ed.), Theoretical models of human development (vol. 1 of Handbook of child psychology, 6th ed., pp. 894-941). Hoboken, NJ: Wiley.

Biddle, S. \& Goudas, M. (1996). Analysis of children's physical activity and its association with 
adult encouragement and social cognitive variables. The Journal of School Health, 66(2), 75-78.

Csikszentmihalyi, M. (1990). Fluir (flow) una psicología de la felicidad (13a ed.). Barcelona: Kairós.

Csikszentmihalyi, M., Rathunde, K. \& Whalen, S. (1993). Talented teenagers: The roots of success and failure. New York: Cambridge University Press.

Dailey, R. (2008). Parental challenge: Developing and validating a measure of how parents challenge their adolescents. Journal of Social and Personal Relationships, 25(4), 643-669. doi:10.1177/0265407508093784

Duda, J. (2013). The conceptual and empirical foundations of Empowering Coaching ${ }^{\mathrm{TM}}$ : Setting the stage for the PAPA project. International Journal of Sport and Exercise Psycchology, 11(4), 37-41. doi:10.1080/1612197X.2013.839414

Dweck, C. (1999). Self-theories: Their role in motivation, personality, and development. Philadelphia: Psychology Press.

Edwardson, C. \& Gorely, T. (2010). Parental influences on different types and intensities of physical activity in youth: A systematic review. Psychology of Sport and Exercise, 11(6), 522-535. doi:10.1016/j.psychsport.2010.05.001

Espasa Calpe (2005). Diccionario de la lengua española. Recuperado de http://www.wordreference.com.

Facio, A., Resett, S., Mistrorigo, C. \& Micocci, F. (2006). Adolescentes argentinos: cómo piensan y sienten. Buenos Aires: Lugar.

Glaser, B. \& Strauss, A. (1967). The Discovery of grounded theory: Strategies for qualitative research. New York: Aldine Publishing Company.

Gute, G., Gute, D., Nakamura, J. \& Csikszentmihalyi, M. (2008). The early lives of highly creative persons: The influence of the complex family. Creativity Research Journal, 20(4), 343-357.

Hektner, J. (2001). Family, school, and community predictors of adolescent growth-conducive experiences: Global and specific approaches. Applied Developmental Science, 5(3), 172-183. doi:10.1207/S1532480XADS0503 5

Hernández Sampieri, R., Fernández-Collado, C. \& Baptista Lucio, P. (2010). Metodología de la investigación (5a ed.). México: McGraw Hill.

Krueger, R. (1991). El grupo de discusión: guía práctica para la investigación aplicada. Madrid: Pirámide.

Lauer, L., Gould, D., Roman, N. \& Pierce, M. (2010). Parental behaviors that affect junior tennis player development. Psychology of Sport and Exercise, 11(6), 487-496. doi:10.1016/j.psychsport.2010.06.008

Lawrence, I. (2009). Soccer and the American dream. s. 1.: University of Stirling. Retrieved from http://hdl.handle.net/1893/2324

Lazarus, R. \& Folkman, S. (1984). Stress, appraisal, and coping. New York: Springer.

Lee, K. (2008). The roles of family styles and adventure program participation on intrinsic motivation. s. 1.: Indiana University.

Lee, M. \& Maclean, S. (1997). Sources of parental pressure among age group swimmers. European Journal of Physical Education, 2(2), 167-177. doi:http://dx.doi. org/10.1080/1740898970020204

Leff, S. \& Hoyle, R. (1995). Young athletes' perceptions of parental support and pressure. Journal of Youth and Adolescence, 24(2), 187-203. doi:10.1007/BF01537149

Marques, S., Pais-Ribeiro, J. \& López, S. (2011). The role of positive psychology constructs in predicting mental health and academic achievement in children and adolescents: a two-year longitudinal study. Journal of Happiness Studies, 12(6), 1049-1062. doi:10.1007/s10902-010-9244-4

Maxwell, J. (2012). Qualitative research design: An interactive approach ( $3 \mathrm{a}$ ed.). Philadelphia: Sage.

Minichiello, C. (2009). El apoyo social percibido: escala de los otros significativos (S.O.S.) adaptada para su uso en población infanto-juvenil. 
Versión argentina. Buenos Aires: Departamento de Publicaciones, Facultad de Psicología, Universidad de Buenos Aires.

Moon, E. (2003). Flow: Family Dynamics and adolescent experiences in soccer. (Doctoral dissertation, Virginia Polytechnic Institute and State University). Retrieved from http://scholar.lib. vt.edu/theses/available/etd-04112003-102110/ unrestricted/Main.pdf

Moreno, J., Cervelló, E. \& González-Cutre, D. (2010). The achievement goal and self-determination theories as predictors of dispositional flow in young athletes. Anales de Psicologia, 26(2), 390-399. Retrieved from http://revistas. um.es/analesps

Muñoz Silva, A. (2005). La familia como contexto de desarrollo infantil: dimensiones de análisis relevantes para la intervención educativa y social. Portularia, 2, 147-163.

Musitu, G., Buelga, S., Lila, M. \& Cava, M. (2004). Adolescencia y familia. Madrid: Síntesis.

Peterson, C. (2013). Pursuing the good life. 100 reflections on positive psychology. New York: Oxford University Press.

Piñuel Raigada, J. (2002). Epistemología, metodología y técnicas del análisis de contenido. Estudios en Sociolinguística, 3(1), 1-42.

Quested, E., Ntoumanis, N., Viladrich, C., Haug, E., Ommundsen, Y., Van Hoye, A., ... Duda, J. (2013). Intentions to drop-out of youth soccer: A test of the basic needs theory among European youth from five countries. International Journal of Sport and Exercise Psycchology, 11(4), 395-407. doi:10.1080/1612197X.2013.830431

Raimundi, M. J., Molina, M. F., Giménez, M. \& Minichiello, C. (2014). ¿Qué es un desafío?: estudio cualitativo de su significado subjetivo en adolescentes de Buenos Aires. Revista Latinoamericana de Ciencias Sociales, Niñez y Juventud, 12(2), 521-534.

Rathunde, K. (2001). Family context and the development of undivided interest: A longitudinal study of family support and challenge and ado- lescents' quality of experience. Applied Developmental Science, 5(3), 158-171. doi:10.1207/ S1532480XADS05034

Rathunde, K., Carroll, M. \& Huang, M. (2000). Families and the forming of children's occupational future. In M. Csikszentmihalyi \& B. Schneider (Eds.), Becoming adult: How teenagers prepare for the world of work. New York: Basic Books.

Rodham, K., Brewer, H., Mistral, W. \& Stallard, P. (2006). Adolescents' perception of risk and challenge: A qualitative study. Journal of Adolescence, 29(2), 261-72. doi:10.1016/j.adolescence. 2005.05.012

Ryan, R. \& Deci, E. (2000). Self-determination theory and the facilitation of intrinsic motivation, social development, and well-being. American Psychologist, 55, 68-78.

Salguero, A., Córdoba, D. \& Sapién, S. (2009). Decisión y planeación de los hijos en el proceso reproductivo de los hombres. Avances en Psicología Latinoamericana, 27(64), 143-153.

Scheuer, N. \& De la Cruz, M. (2010). Las ideas de los niños sobre el aprendizaje: conocerlas y potenciarlas. Aula de Innovación Educativa, 190, 41-44. Retrieved from https:// c51888e0-a-62cb3a1a-s-sites.googlegroups. com/site/gisistemasrepresentacion/home/ archivos/OE_D_2010Scheuer y de la Cruz. pdf?attachauth=ANoY7cr QA5SVDln126D8ZYM1Q0PPvO91BXIQMYvH79hg_sij9ca6nkIkJ WnMaV81NjHQj7XyuN9GSDMD6V3V5SqFWwyXph111jDxdVzzF7vB0OHQ6d-PVomRNcA6V0sBbPKgnQ4Uv3XBs1SA6qWEAITMQuZYQthfZGujIlAnPQ540whgyTsOHw9hIxTms soSjTYrc8NYyBe-w_JGn8tBThBmuWO-S4pNG2mkRakDxpEgAiTeFz2ZpqMX5usoDW6nLb97aEZgOxe_IbSYfzLiIOjjjgPJbFA $==$ \&attredirects $=0$ Schmidt, J. \& Padilla, B. (2003). Self-esteem and family challenge: An investigation of their effects on achievement. Journal of Youth and Adolescence, 32(1), 37-46. doi:10.1023/A:1021080323230 
Schmidt, V., Maglio, A. L., Messoulam, N., Molina, M. F. \& González, M. A. (2010). La comunicación del adolescente con sus padres: construcción y validación de una escala desde un enfoque mixto. Interamerican Journal of Psychology, 44(2), 299-311.

Schmidt, V., Marconi, A., Messoulam, N., Maglio, A., Molina, M. \& González, M. (2008). La comunicación entre padres e hijos desde la percepción adolescente. Una aproximación etnopsicológica. Revista de Psicología Social Aplicada, 17(1), 1-23.

Sinnott, J. (2013). Positive Psychology. Advances in understanding adult motivation. Zhurnal Eksperimental'noi i Teoreticheskoi Fiziki. New York: Springer. Retrieved from http://scholar. google.com/scholar?hl=en\&btnG=Search\&q=intitle:No+Title\#0

Strauss, A. \& Corbin, J. (1990). Basics of qualitative research: Grounded Theory procedures and techniques. Philadelphia: Sage.
Suárez Relinque, C., del Moral Arroyo, G. \& González Fernández, M. (2013). Consejos prácticos para escribir un artículo cualitativo publicable en Psicología. Psychosocial Intervention, 22(1), 71-79. doi:10.5093/in2013a9

Torregrosa, M., Cruz, J., Sousa, C., Viladrich, C., Villamarín, F., García-Mas, A. \& Palou, P. (2007). La influencia de padres y madres en el compromiso deportivo de futbolistas jóvenes. Revista Latinoamericana de Psicología, 39(2), 227-237.

Velandia Morales, A. (2008). Investigación cualitativa y psicología del consumidor: alternativas de aplicación. Avances en Psicología Latinoamericana, 26(2), 290-303.

Zalta, A. \& Chambless, D. (2011). Testing a developmental model of anxiety with the Parental Facilitation of Mastery Scale. Journal of Anxiety Disorders, 25(3), 352-61. doi:10.1016/j.janxdis.2010.10.009

\section{Fecha de recepción: 3 de febrero de 2014 fecha de aceptación: 30 de septiembre de 2014}

